\title{
A Comparison of Modelling Strategies for Value-Added Analyses of Educational Data
}

\author{
Neil H. Spencer ${ }^{1}$ and Antony Fielding ${ }^{2}$ \\ ${ }^{1}$ Department of Statistics, Accounting and Management Systems, \\ University of Hertfordshire, Hertford Campus, Mangrove Road, \\ Hertford, SG13 8QF, UK. \\ ${ }^{2}$ Department of Economics, University of Birmingham, Edgbaston, \\ Birmingham, B15 2TT, UK.
}

\section{Summary}

Modelling strategies for value-added multilevel models are examined. These types of models typically include an endogenous variable and this causes difficulties for the standard estimation techniques that are commonly used to analyse multilevel models. Two alternative estimation strategies are proposed: one using an instrumental variable approach and the other using a Bayesian analysis as available through the BUGS software. We conclude that the approach offered by the BUGS software has advantages over more classical estimation methods.

Keywords: Hierarchical Modelling; Iterative Generalized Least Squares; Gibbs Sampling; Endogeneity. 


\section{Introduction}

Since the mid 1980s, the analysis of hierarchically structured data through estimation of random effects multilevel models has become commonplace. A variety of procedures have been developed and algorithms implemented in readily available software (VARCL (Longford, 1988), HLM (Bryk et al, 1996), Proc Mixed from SAS (SAS Institute, 1992), MLn and MLwiN (Rasbash and Woodhouse, 1995; Goldstein et al, 1998)). The MLn and MLwiN software we use in this paper is developed around the Iterative Generalised Least Squares (IGLS) methods of Goldstein (1995). More recently, Markov Chain Monte Carlo (MCMC) methods of carrying out modelling in a Bayesian framework have increased in popularity, implementing Gibbs and other sampling techniques. Improvements in technology have meant that these computer intensive approaches to modelling have become more accessible to researchers, and the development of the BUGS software package (Spiegelhalter et al, 1995a) has further increased interest in such modelling strategies. A good account of the methodology surrounding these procedures is provided by Draper (1998).

In this paper we discuss a multilevel model which is an example of a common situation where classical assumptions of independence of explanatory variables and the random effects no longer holds. The IGLS procedures available in MLwiN will in this case not directly yield consistent estimators. However, adaptations based on instrumental variable (IV) methods to produce a consistent estimation procedure have been suggested by Fielding and Spencer (1997). This is available as a MLwiN macro IV (http://www.bham.ac.uk/economics/staff/tony.htm). Here we discuss a case where the Bayesian modelling strategy through the MCMC methods of the BUGS software may be preferable in many regards. It should be noted, however, that the recently available update of MLn, called MLwiN, now also has the capability to carry out some Bayesian modelling. Further work comparing the BUGS and MLwiN approaches is planned.

Below, we present the dataset and model used in this paper, and then contrast results from the alternative estimation strategies. We then present results obtained using simulated datasets and consider different parameterisations of the scale matrices used in BUGS. Conclusions and ideas for further work are presented. 


\section{Dataset and Model}

We consider a hierarchical dataset consisting of data collected on 1946 pupils from 14 educational establishments in England. The response variable considered, $\mathrm{y}_{\mathrm{ij}}$, is a standardised points score in General Certificate of Education Advanced Level (A-level) for pupil $j$ in establishment $i$. This is a measure of academic success over usually two, three or four examinations normally taken when aged approximately 18 years. Explanatory variables are GCSE points score (a measure of academic success over several subject areas in the British "General Certificate of Secondary Education", normally taken when aged approximately 16 years), age and gender for a pupil.

More specifically, the model we consider is

$$
\mathrm{y}_{\mathrm{ij}}=\beta_{0}+\beta_{1} \mathrm{x}_{1 \mathrm{ij}}+\beta_{2} \mathrm{x}_{2 \mathrm{ij}}+\beta_{3} \mathrm{x}_{3 \mathrm{ij}}+\mathrm{u}_{\mathrm{i}}+\varepsilon_{\mathrm{ij}}
$$

where, $x_{1 i j}$ is the standardised GCSE points score, $x_{2 i j}$ is the pupil's age and $\mathrm{x}_{3 \mathrm{ij}}$ is a binary variable indicating gender. The $\mathrm{u}_{\mathrm{i}}$ parameter is a random effect varying between schools, with variance $\sigma_{\mathrm{u}}^{2}$, and $\varepsilon_{\mathrm{ij}}$ is the usual pupil-level random effect, with variance $\sigma_{\varepsilon}^{2}$.

It is deliberately simple in form as the object of the work presented here is to compare modelling approaches rather than draw substantive conclusions from the data. It also serves to remind the reader that models need not be complex in order to be problematic.

Despite its simplicity, this model is of considerable interest in school effectiveness work. By controlling for the GCSE points score and other relevant factors for a pupil when analysing the A-level points score, the study can examine the "value added" by a school. This is usually evaluated by estimation of the school random effects or "residuals". This form of model is of the same genre as that used by Gray et al (1995) and Goldstein and Thomas (1996). 


\section{The Problem of Endogeneity}

Estimates of the parameters of the model shown above can be easily obtained using standard multilevel modelling packages. However, this approach ignores the fact that the GCSE point score may not be independent of the random effects in the model which such estimation requires for good properties. Indeed this variable may be regarded as endogenous and could itself be modelled using the same data:

$$
\mathrm{x}_{1 \mathrm{ij}}=\gamma_{0}+\gamma_{2} \mathrm{x}_{2 \mathrm{ij}}+\gamma_{3} \mathrm{x}_{3 \mathrm{ij}}+\mathrm{v}_{\mathrm{i}}+\mathrm{e}_{\mathrm{ij}}
$$

where $\mathrm{x}_{1 \mathrm{ij}}, \mathrm{x}_{2 \mathrm{ij}}$ and $\mathrm{x}_{3 \mathrm{ij}}$ are as above, $\mathrm{v}_{\mathrm{i}}$ is a random effect varying between schools and $\mathrm{e}_{\mathrm{ij}}$ is the pupil- level random effect. The $\mathrm{u}_{\mathrm{i}}$ in the model for $\mathrm{y}_{\mathrm{ij}}$ and the $\mathrm{v}_{\mathrm{i}}$ may both be regarded as school effects. It could therefore be argued that we may encounter estimation difficulties due to the correlation between $\mathrm{x}_{1 \mathrm{ij}}$ and the school random effect in the A-level model through the $\mathrm{v}_{\mathrm{i}}$. However, the extent to which this correlation exists is open to question as most of the students did not study for their A-level examinations at the same establishment at which they studied for their GCSE examinations. The $v_{i}$ could then be regarded as exogenous for the A-level model. For situations where correlations at this level of the data structure may be problematic, Rice et al (1998) have developed strategies known as conditional IGLS.

Of prime concern in this paper is the correlation that exists between $\mathrm{X}_{1 \mathrm{ij}}$ and the random part of the A-level model through the pupil-specific random terms $\varepsilon_{\mathrm{ij}}$ and $\mathrm{e}_{\mathrm{ij}}$. Both these terms contain unexplained and unmeasurable pupil factors and thus cause $\mathrm{x}_{1 \mathrm{ij}}$ to be endogenous for the A-level model.

This endogeneity means that the assumption of independence of regressors and model error cannot be sustained and thus the parameter estimates obtained by a direct MLwiN analysis may be inconsistent. The degree to which this inconsistency affects the estimates is governed by the size of the covariance between the pupil effects at GCSE and A-level. As these covariances are not known at the time of the analysis, some adjustment of the estimation process must be undertaken to ensure that consistent estimates are produced. As the endogeneity is caused by correlations at the lowest level of the data hierarchy, the conditional IGLS methods of Rice et al (1998) are not available. 


\section{Consistent Estimation using Instrumental Variables}

A method for obtaining consistent parameter estimates for models such as those considered in this paper is given in Fielding and Spencer (1997). An instrument set is created using the regressors in the A-level model with the endogenous regressor (the GCSE score) being replaced by an instrumental variable (IV). This instrumental variable is obtained by a multilevel modelling of the GCSE score using variables that are assumed to be independent of the school and pupil effects in the A-level model. Here, we use dummy variables that define whether the Alevels taken by the student are all science/mathematical subjects, all non science/mathematical subjects or a mixture. In order to create an instrumental variable that is a good predictor of GCSE score, it would be preferable to have a number of regressors that are relatively unrelated to the A-levels. However, further regressors of this type are not available from the study and this raises the issue of the collection of good relevant data in future studies so that this IV procedure can be carried out more efficiently.

Once the estimation of the parameters of the GCSE model has taken place, estimates of the GCSE score are obtained using just the fixed part of the model (i.e. ignoring any school and pupil effects) and these estimates are used in place of the original GCSE score in the instrument set. There is a danger of introducing correlations between the instrument set and the random part of the A-level model if any of the random effects are used to produce estimates of the GCSE score so we choose here to only use the fixed part of the model. If the regressors used in the GCSE model are truly independent of the school and pupil effects in the Alevel model, then the estimate of the GCSE score from the fixed part of the model will be as well. The instrument set is then independent of the disturbance of the Alevel model, and consistent estimates of the parameters of the A-level model can be obtained using standard IV techniques.

The Iterative Generalized Least Squares algorithm (used in MLwiN) can then be used to obtain estimates of the random parameters of the A-level model, with the fixed parameters being constrained to those obtained by the IV estimation. These random parameters can then be used to obtain standard errors for the IV estimates.

It could be argued that the dummy variables used to construct the instrument for the GCSE score may not be independent of the school and pupil effects in the Alevel model. This problem of selecting appropriate regressors for the modelling of the GCSE score is an essential problem with the instrumental variable method of obtaining consistent parameter estimates. All that can be said regarding this 
problem here is that the resulting instrument is likely to be less correlated with the disturbance of the A-level model than the original GCSE score, and the problems of inconsistency are thus likely to be less.

Table 1 shows the results of estimating the parameters of the A- level model using MLwiN with and without the IV method of estimation. It appears from these results that inconsistency of a standard analysis does not present a serious problem for this example. However, in general it is not always the case that reliable estimates are obtained and later we shall see a case where they are not. As a general rule we may prefer the IV estimators since they will suffer less from any consistency problems.

Table 1: Results

\begin{tabular}{lclllll}
\hline Parameter & \multicolumn{2}{c}{ Without IV } & \multicolumn{2}{c}{ With IV } & \multicolumn{2}{c}{ BUGS } \\
\hline$\beta_{0}$ & 0.0236 & $(0.0551)$ & 0.0097 & $(0.0667)$ & 0.0363 & $(0.1241)$ \\
$\beta_{1}$ & 0.5735 & $(0.0210)$ & 0.5116 & $(0.0903)$ & 0.5453 & $(0.2188)$ \\
$\beta_{2}$ & 0.0775 & $(0.0196)$ & 0.0729 & $(0.0268)$ & 0.0747 & $(0.0339)$ \\
$\beta_{3}$ & -0.0185 & $(0.0383)$ & -0.0180 & $(0.0403)$ & -0.0158 & $(0.0500)$ \\
$\sigma_{\varepsilon}^{2}$ & 0.6936 & $(0.0223)$ & 0.6972 & $(0.0272)$ & 0.7731 & $(0.0246)$ \\
$\sigma_{u}^{2}$ & 0.0261 & $(0.0132)$ & 0.0236 & $(0.0122)$ & 0.4833 & $(0.2038)$ \\
\hline
\end{tabular}

Of course, as may be seen from table 1, the standard errors obtained from the IV estimation procedure are larger, but not much larger, than those obtained from straightforward IGLS. The general problem of large standard errors and implied efficiency is well known. However, in many instances, as in these results, acceptable results can be obtained (see Spencer, 1998 for a fuller discussion of this problem).

\section{Modelling with BUGS}

The advantage of a Bayesian approach to the modelling of the data, as implemented in BUGS, is that the endogeneity of the GCSE points score, described above, can be built into the model. To show this, a directed graph can be created (see figure 1). In the graph, rectangles denote constants fixed by the study design (age, gender). Nodes with circles/ovals around them are stochastic variables that are given a distribution and may be data (e.g. GCSE points score, A-level points score) or parameters (e.g. $\beta_{0}, \gamma_{0}$ ). Dashed arrows linking nodes indicate that there is a local function linking the parent nodes (at the tails of the 
arrows) to the child nodes (at the heads of the arrows). Solid arrows indicate that a stochastic dependence exists. As well as being an alternative way to display the model being used, this graph can be used as an aid to write the model in the BUGS language.

\section{Figure 1: Directed Graph}

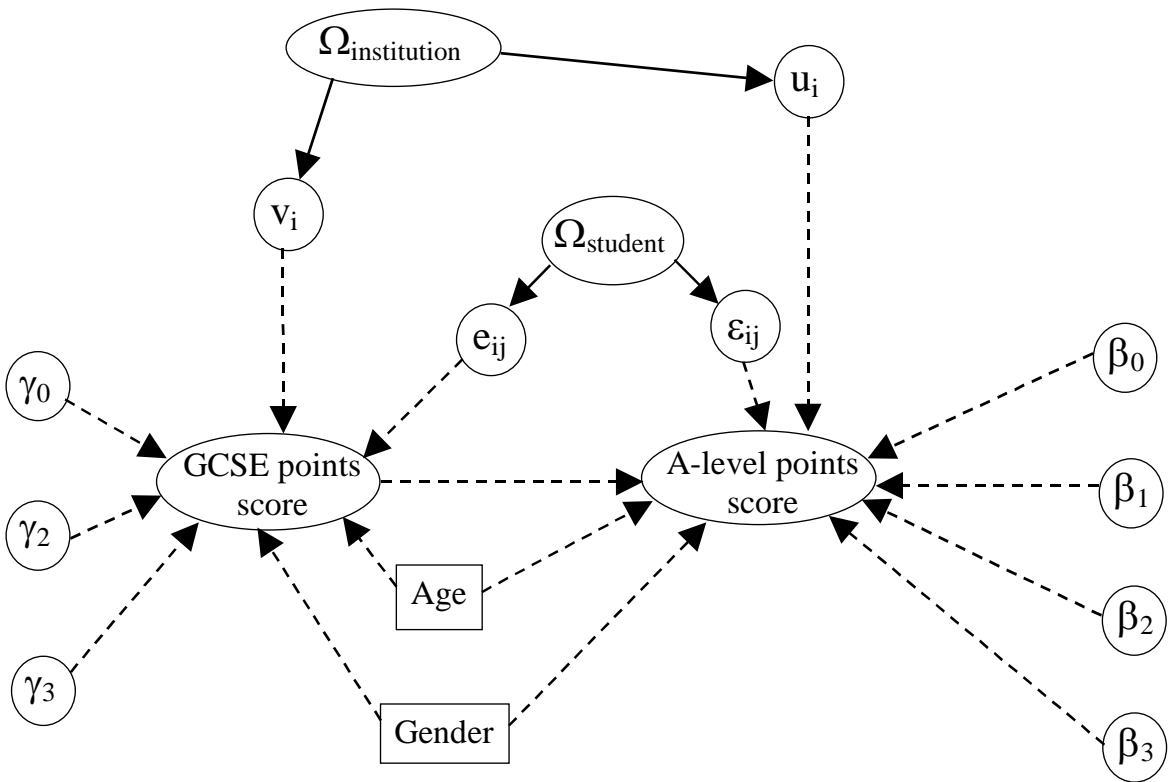

Using the directed graph, we define the A-level score to be dependent on the GCSE score, age and gender (the fixed part of the A-level model). We also simultaneously define the GCSE score to be dependent on age and gender (the fixed part of the model above). Both the A-level score and GCSE score have school effects associated with them. To define these on our directed graph, we have created two effects $\left(\mathrm{u}_{\mathrm{i}}\right.$ associated with the A-level score and $\mathrm{v}_{\mathrm{i}}$ associated with the GCSE score) coming from a bivariate normal distribution. To define two pupil effects, we have the A-level score and GCSE score coming from a bivariate normal distribution. It is these bivariate normal distributions (which have nonzero covariances associated with them) which induce endogeneity.

The basic idea behind the MCMC techniques is that instead of carrying out complex calculations to arrive at an exact or approximate estimate of a quantity of interest from the model, series of simulated values are obtained. The idea is that these simulated values are eventually (once the iteration process has converged) coming from the distribution appropriate for the quantity of interest. If this is the 
case then the mean, standard deviation, etc. of the posterior distribution of the quantity of interest can be examined using the simulated values. Gibbs sampling is a technique for obtaining MCMC simulations where, sequentially, each parameter of interest is simulated from its conditional distribution given the most recent updates available of the other parameters. For more details of MCMC and Gibbs sampling techniques, see texts such as Draper (1998), Smith and Roberts (1993).

\section{Assigning Priors}

A frequent criticism of Bayesian approaches to statistical analyses is that concerning the need to assume prior distributions for the parameters. In the analysis presented here, we use non-informative priors. For the fixed effect coefficients, we use Normal distributions with means of zero and precisions of $0 \cdot 00001$. For the random parameters, we need to define two bivariate normal distributions. Each of these distributions has a vector of means associated with it and we define these to be zeros. Each distribution also has a 2 dimensional covariance matrix associated with it, and the precision of the covariance matrix is assumed to have a Wishart distribution. This Wishart distribution has an associated 2 dimensional scale matrix and degrees of freedom chosen to be as small as possible (the rank of the bivariate normal distribution: 2) to represent vague prior knowledge (Spiegelhalter et al, 1995b). The difficulty comes when having to define the scale matrix and is investigated in later, but for now we proceed by defining an arbitrary scale matrix with the numbers 2 on the main diagonal and 1 in the off-diagonal positions. See Spiegelhalter et al (1995a) for more discussion on this issue.

\section{Burn-in Iterations and Convergence}

In order to obtain results from BUGS, the MCMC iterations begin with pre-set starting values and a number of "burn-in" iterations are required until the parameter realisations obtained by the Gibbs sampling are not influenced by these starting values and have converged so that they come from stationary distributions.

One way of deciding on the number of "burn-in" iterations that need to take place is to look at a trace plot of the estimates of a parameter against the iteration number. Figure 2 shows an example of a trace plot in which the parameter estimates appear to be exhibiting a pattern of random fluctuations around a steady mean after only a very small number of iterations. Here, very few "burn-in" iterations are required. Another often viewed pattern for a trace plot is one where the parameter estimates take a larger number of iterations before the trace plot 
9

takes on the appearance of figure 2. In this latter case, all the iterations before the parameter estimates take the form of random fluctuations around a steady mean are regarded as "burn-in" iterations.

Diagnostic statistics such as those proposed by Geweke (1992), Gelman and Rubin (1992), Raftery and Lewis (1992), Heidelberger and Welch (1983) can also be used to aid determination of the number of "burn-in" iterations required.

The trace plots and diagnostic statistics can be obtained from a BUGS analysis with the help of a suite of menu-driven S-Plus functions known collectively as CODA (Best et al, 1995) that accompanies the BUGS software.

Figure 2: Trace Plot

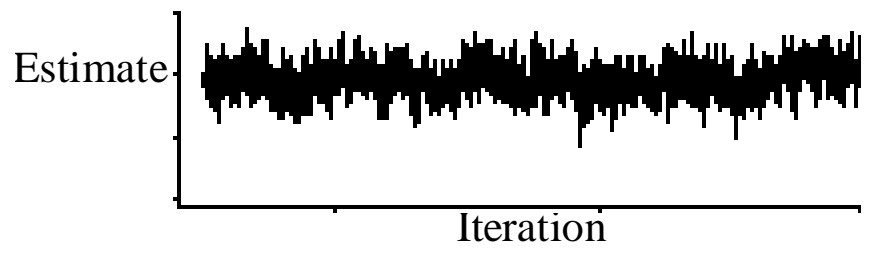

\section{BUGS Results}

Table 1 shows the results obtained from a BUGS analysis of the data described in above with a large burn-in of 5000 iterations and 5000 monitored iterations. The results obtained again indicate that the estimates produced by the initial MLwiN analysis are acceptable. The BUGS estimates of the fixed effects are similar to those produced by MLwiN, but have higher standard errors. The estimates of the variances of the random effects are larger than those produced by MLwiN, particularly for the school level random effect. This may be in part due to difficulties in obtaining an estimate of a variance which is near the lower limit of zero.

In this example data it appears that all three methods used give comparable results. We have already commented on general reasons why we might prefer IV methods to standard IGLS. However, given that MCMC modelling is more complicated to implement, it appears to convey few advantages for the case discussed, particularly in view of the lower precision of the results. Below we shall see, however, that the MCMC approach can have advantages when the endogeneity issue has more impact on the standard IGLS estimation procedure. 


\section{Simulated Data}

In this section, we explore the issue further by means of simulated datasets, each with a similar structure to that defined by equations (1) and (2). The parameter values that the simulations are based on come from the BUGS analysis discussed above: $\beta_{0}=0.0363, \beta_{1}=0.5453, \beta_{2}=0.0747, \beta_{3}=-0.0158, \sigma_{\varepsilon}^{2}=0.7731$, $\sigma_{\mathrm{u}}^{2}=0.4833, \gamma_{0}=-1.1419, \gamma_{2}=0.1346, \gamma_{3}=-0.1221, \sigma_{\mathrm{e}}^{2}=0.9533, \sigma_{\mathrm{v}}^{2}=$ $0.2252, \sigma_{\varepsilon \mathrm{e}}=0.4462, \sigma_{\mathrm{uv}}=0.0689$, where $\sigma_{\varepsilon \mathrm{e}}$ is the covariance between $\varepsilon_{\mathrm{ij}}$ and $\mathrm{e}_{\mathrm{ij}}$ and $\sigma_{\mathrm{uv}}$ is the covariance between $\mathrm{u}_{\mathrm{i}}$ and $\mathrm{v}_{\mathrm{i}}$. Results obtained using parameter values from the MLwiN with or without the IV procedure could have been presented here. However, these parameter values are similar in scale and nature to the BUGS ones used here and yield similar results. They are thus not presented here for the sake of brevity. The authors' experience with other datasets and models, not closely related to those shown in this paper but having similar endogeneity problems, also yield similar conclusions.

Table 2 shows the mean estimate for each parameter over the fifty datasets obtained using each estimation method, together with the associated empirical standard deviation.

Table 2: Simulation Results

\begin{tabular}{|c|c|c|c|c|c|c|}
\hline \multirow[b]{2}{*}{ Parameter } & \multirow{2}{*}{$\begin{array}{l}\text { Target } \\
\text { Values }\end{array}$} & & \multicolumn{2}{|c|}{ MLwiN } & \multirow{2}{*}{\multicolumn{2}{|c|}{ BUGS }} \\
\hline & & & Without IV & With IV & & \\
\hline$\beta_{0}$ & $0 \cdot 0363$ & & $0 \cdot 1210(0 \cdot 2066)$ & $0 \cdot 2250(1 \cdot 159)$ & $0 \cdot 0408$ & $(0 \cdot 2402)$ \\
\hline$\beta_{1}$ & $0 \cdot 5453$ & & $1 \cdot 010 \quad(0.0242)$ & $0.4628(9 \cdot 592)$ & $0 \cdot 6682$ & $(0 \cdot 4770)$ \\
\hline$\beta_{2}$ & 0.0747 & & $0 \cdot 1245(0.0287)$ & $0.0045(1.422)$ & $0 \cdot 0891$ & $(0.0631)$ \\
\hline$\beta_{3}$ & $-0 \cdot 0158$ & - & $0.0723(0.0531)$ & $0 \cdot 2153(2 \cdot 077)$ & $-0 \cdot 0141$ & $(0 \cdot 1251)$ \\
\hline$\sigma_{\varepsilon}^{2}$ & 0.7731 & & $0.4669(0.1753)$ & $92 \cdot 31 \quad(403 \cdot 4)$ & 0.9640 & $(0 \cdot 0512)$ \\
\hline$\sigma_{u}^{2}$ & $0 \cdot 4833$ & & $0.5571(0.0290)$ & $20 \cdot 16 \quad(84 \cdot 33)$ & $0 \cdot 4146$ & $(0 \cdot 0717)$ \\
\hline
\end{tabular}

From table 2, we see that we have a situation where the MLwiN results without the instrumental variable procedure appear to be suffering from problems of inconsistency. The mean estimate of $\beta_{1}$ is well over 2 empirical standard deviations from the target value and it is this pattern of an inflated estimate for the 
11

coefficient of the endogenous variable that is typical when no adjustment for the endogeneity is made. This is because the positive correlation between the GCSE score and the random part of the A-level model causes a positive bias in the estimate of the coefficient.

When the instrumental variables procedure is employed, it is apparent that although we may well be obtaining consistent estimates, the large standard errors mean that the usefulness of the results is limited. This pattern of large standard errors is a feature of the instrumental variables procedure when the instrument constructed is not a good predictor of the endogenous variable. The results obtained by BUGS are more useful with lower standard errors being produced and the mean parameter estimates being relatively near the target values. The only difficulty apparently being experienced is with the estimate of $\sigma_{\mathrm{e}}^{2}$ which is slightly higher than we would wish, with a relatively small standard error.

\section{Alternative Scale Matrices}

Above, we stated that we were able to use non-informative priors for the BUGS analysis, but still had to define scale matrices associated with the covariance matrices of the school and pupil effects. In this section, we investigate the sensitivity of the parameter estimates to changes in the scale matrices.

The first parameterisation of the scale matrix considered here has the number 1 on the main diagonal and 0.5 in the off-diagonal positions. Scale parameterisation two is the identity matrix. Scale parameterisation three has 0.7731 and 0.9533 on the main diagonal and 0.4462 in the off-diagonal positions for the pupil effects (the A-level and GCSE pupil level variances that the above simulations are based on) and for the school effects has 0.4833 and 0.2252 on the main diagonal and 0.0689 in the off-diagonal positions (the A-level and GCSE school level variances that the simulations are based on). Scale parameterisation four has 100 on the main diagonal and 10 in the off-diagonal positions for both the pupil and school effects.

$\underline{\text { Table 3: Results from Alternative Scale Parameterisation }}$

\begin{tabular}{lccccc} 
& Target & \multicolumn{4}{c}{ Scale Parameterisation } \\
\hline Parameter & Values & 1 & 2 & 3 & 4 \\
\hline$\beta_{0}$ & 0.0363 & 0.0427 & 0.0395 & 0.0423 & -1.426 \\
& & $(0.2413)$ & $(0.2414)$ & $(0.2423)$ & $(0.6555)$ \\
$\beta_{1}$ & 0.5453 & 0.6673 & 0.6512 & 0.6584 & -1.954
\end{tabular}




\begin{tabular}{|c|c|c|c|c|c|}
\hline & & $\begin{array}{r}12 \\
(0.4884)\end{array}$ & $(0.4856)$ & $(0 \cdot 4968)$ & $(1.420)$ \\
\hline$\beta_{2}$ & 0.0747 & $\begin{array}{c}0.0893 \\
(0 \cdot 0648)\end{array}$ & $\begin{array}{c}0.0872 \\
(0 \cdot 0645)\end{array}$ & $\begin{array}{c}0.0884 \\
(0.0661)\end{array}$ & $\begin{array}{l}-0 \cdot 2168 \\
(0 \cdot 2038)\end{array}$ \\
\hline$\beta_{3}$ & -0.0158 & $\begin{array}{l}-0 \cdot 0141 \\
(0 \cdot 1268)\end{array}$ & $\begin{array}{l}-0.0117 \\
(0.1270)\end{array}$ & $\begin{array}{l}-0.0128 \\
(0.1284)\end{array}$ & $\begin{array}{c}0.4349 \\
(0.3942)\end{array}$ \\
\hline$\sigma_{\varepsilon}^{2}$ & 0.7731 & $\begin{array}{c}0.9626 \\
(0.0512)\end{array}$ & $\begin{array}{c}0.9626 \\
(0.0512)\end{array}$ & $\begin{array}{c}0.9627 \\
(0.0512)\end{array}$ & $\begin{array}{c}1 \cdot 108 \\
(0 \cdot 0513)\end{array}$ \\
\hline$\sigma_{u}^{2}$ & 0.4833 & $\begin{array}{c}0 \cdot 3297 \\
(0 \cdot 0711)\end{array}$ & $\begin{array}{r}0.3304 \\
(0.0710)\end{array}$ & $\begin{array}{c}0.2652 \\
(0.0707)\end{array}$ & $\begin{array}{c}8 \cdot 784 \\
(0 \cdot 5490)\end{array}$ \\
\hline
\end{tabular}

Table 3 gives the results from analysing the 50 simulated datasets used above. As can be seen, the mean estimates are quite stable for parameterisations 1, 2 and 3 and that used in table 2. This is a good sign in that it appears that the results are fairly robust to the choice of scale matrices. Parameterisation 4, with the extreme scale matrices, produces worse results. As in table 2, the mean estimates for the variances are less satisfactory than those for the fixed effect coefficients.

\section{Conclusions and Further Work}

In this paper, we have shown that a BUGS analysis which has the endogeneity of the GCSE score built into the model structure can produce results which are clearly preferable to a classical analysis which ignores the endogeneity problem. The BUGS analysis can also be preferable to an analysis using instrumental variable methods which may produce estimates with unacceptably large standard errors. Also with the endogeneity being properly modelled, assumptions do not have to be made regarding the independence of the instrumental variable and the disturbance in the A-level model. It has been shown that non-informative priors can be used and that the results appear to be robust to different choices of scale matrices, providing these choices are reasonable.

More work is needed to identify those occasions, such as demonstrated in table 1, where a classical analysis can produce acceptable results despite endogeneity concerns. Additionally, further research is needed regarding the BUGS estimates of the variances in the model and how they can be improved. Nevertheless, despite these areas of research that need more investigation, it is clear from this paper that the BUGS approach to modelling can give distinct advantages over more classical approaches. 


\section{References}

Best, N.G., Cowles, M.K. and Vines, S.K. (1995), CODA Manual, Version 0.30, Cambridge: MRC Biostatistics Unit.

Bryk, A.S., Raudenbush, S.W. and Congdon, R.T. (1996), HLM: Hierarchical Linear Modelling with the HLM/2L and HLM/3L Programs, Chicago: Scientific Software International.

Draper, D. (1998), Bayesian Hierarchical Modeling http://www.bath.ac.uk/ $\sim$ masdd/.

Fielding, A. and Spencer, N.H. (1997), "Modelling cost-effectiveness in general certificate of education A level: Some practical methodological innovations" in C.E. Minder and H. Friedl (eds.) Good Statistical Practice: Proceedings of the 12th International Workshop on Statistical Modelling, Biel/Bienne, July 7 to 11, 1997, Vienna: Schriftenreihe der Österreichischen Statistischen Gesellschaft.

Gelman, A. and Rubin, D.B. (1992), "Inference from iterative simulation using multiple sequences”. Statistical Science 7, 457-472.

Geweke, J. (1992), "Evaluating the accuracy of sampling-based approaches to calculating posterior moments" in J.M. Bernardo, J.O. Berger, A.P. Dawid and A.F.M. Smith (eds.) Bayesian Statistics 4, Oxford: Clarendon Press.

Goldstein, H. (1995), Multilevel Statistical Models, London: Edward Arnold.

Goldstein, H., Rasbash, J., Plewis, I., Draper, D., Browne, W., Yang, M., Woodhouse, G. and Healy, M. (1998), A User's Guide to MLwiN, London: Institute of Education, University of London.

Goldstein, H. and Thomas, S. (1996), "Using examination results as indicators of school and college performance", Journal of the Royal Statistical Society, Series A 159, 407-442.

Gray, J., Jesson, D., Goldstein, H., Hedger, K. and Rasbash, J. (1995), “A multilevel analysis of school improvement: changes in schools' performance over time”, School Effectiveness and School Improvement 6, 97-114.

Heidelberger, P. and Welch, P. (1983), "Simulation run length control in the presence of an initial transient”, Operations Research 31, 1109-1144. 
Longford, N.T. (1988), VARCL -- software for variance component analysis of data with hierarchically nested random effects (maximum likelihood), Princeton, NJ: Education Testing Service.

Raftery, A.L. and Lewis, S. (1992), "How many iterations in the Gibbs sampler?" in J.M. Bernardo, J.O. Berger, A.P. Dawid and A.F.M. Smith (eds.) Bayesian Statistics 4, Oxford: Clarendon Press.

Rasbash, J. and Woodhouse, G. (1995), MLn Command Reference, Version 1.0, London: Institute of Education, University of London.

Rice, N., Jones, A. and Goldstein, H. (1998), "Multilevel models where the random effects are correlated with the fixed predictors: a conditioned iterative generalised least squares estimator (CIGLS)”. Multilevel Modelling Newsletter 10(1), pp 7-11.

SAS Institute (1992), SAS Technical Report P-229, SAS/STAT Software: Changes and Enhancements, Cary, NC: SAS Institute, Inc.

Smith, A.F.M. and Roberts, G.O. (1993), "Bayesian computation via the Gibbs sampler and related Markov chain Monte Carlo methods", Journal of the Royal Statistical Society, Series B 55, 3-23.

Spencer, N.H. (1998), “Consistent parameter estimation for lagged multilevel models", University of Hertfordshire Business School Statistics Technical Report 1, UHBS 1998:19.

Spiegelhalter, D.J., Thomas, A., Best, N.G. and Gilks, W.R. (1995a), BUGS: Bayesian inference Using Gibbs Sampling, Version 0.50, Cambridge: MRC Biostatistics Unit.

Spiegelhalter, D.J., Thomas, A., Best, N.G. and Gilks, W.R. (1995b), BUGS: Examples Volume 2, Version 0.50, Cambridge: MRC Biostatistics Unit. 